\title{
HUMEDALES ARTIFICIALES PARA EL DESARROLLO COMUNITARIO: EL CASO DE UNA COMUNIDAD MAZAHUA DE MÉXICO
}

\section{Artificial Wetlands for Community Development: the Case of a Mazahua Community in Mexico}

\section{Daniel Granados Laudino}

Instituto de Ciencias Agropecuarias y Rurales, Universidad Autónoma del Estado de México, El Cerrillo Piedras Blancas, Toluca, Estado de México

C.P. 50090 Correo-e: danielgl.24@hotmail.com

\section{Acela Montes de Oca Hernández}

Centro de Investigaciones en Ciencias Sociales y

Humanidades, Universidad Autónoma del Estado

de México Correo-e: amhplata2007@yahoo.com.mx

\section{Sergio Moctezuma Pérez}

Instituto de Ciencias Agropecuarias y Rurales,

Universidad Autónoma del Estado de México,

Autor para correspondencia

Correo-e: smoctezumap@uaemex.mx

Recibido: 6/5/2019 • Aprobado: 30/5/2019

Cómo citar: Granados Laudino, D., Montes de Oca Hernández, A., \& Moctezuma Pérez, S. (2019). Humedales artificiales para el desarrollo comunitario: el caso de una comunidad Mazahua de México. Ciencia, Ambiente y Clima, 2(1), 35-43. doi: https://doi. org/10.22206/cac.2019.v2i1.pp35-43

\section{Resumen}

Los humedales artificiales son sistemas socio-ecológicos que proporcionan beneficios ambientales, sociales y económicos a los grupos humanos que los rodean. Los humedales se componen de elementos naturales como el agua, la vegetación, el suelo, entre otros. Además, de elementos humanos como la fuerza de trabajo humano, para su mantenimiento, la organización social, necesaria para el aprovechamiento de los beneficios, y de reglas de operación sobre el uso y manejo del agua. En este artículo los autores analizamos el caso de un humedal artificial localizado en una comunidad de origen indígena del centro de México. Este humedal existe desde hace dos décadas y fue construido por iniciativa de un grupo de indígenas mazahuas y una fundación orientada al mejoramiento de las sociedades vulnerables. Para realizar la investigación que sustenta este artículo se utilizó una metodología cualitativa, apoyada en la técnica de la entrevista. Los resultados de la investigación indican que la relación entre la fundación y la comunidad indígena fue exitosa, debido a que la creación y operación del humedal artificial es un ejemplo de la confianza, reciprocidad e interés por alcanzar un desarrollo local.

Palabras clave: antropología; etnografía; sociedades indígenas; organización social; reciprocidad.

\section{Abstract}

Artificial wetlands are socio-ecological systems that provide environmental, social and economic benefits to the human groups that surround them. Wetlands are composed of natural elements such as water, vegetation, soil, and others. Also, wetlands are composed of human elements, such as, the work force for their maintenance, the social organization necessary to take advantage of the benefits as well as the rules of operation on the use and management of water. In this article, the authors analyze the case of an artificial wetland located in an indigenous community in central Mexico. This wetland has existed for two decades and was built by the initiative of a group of indigenous Mazahuas and a foundation aimed at the improvement of vulnerable societies. To conduct the research that supports this article, a qualitative methodology was used, which was based on the interview technique. The results of the research indicate that the relationship between the foundation and the indigenous community was successful, because the creation and operation of the artificial wetland is the result of trust, reciprocity and interest between both actors to achieve local development.

Keywords: Anthropology; ethnography; indigenous societies; social organization; reciprocity. 


\section{Introducción}

Los humedales son sistemas socio-ecológicos debido a la interacción entre sus componentes naturales y los beneficios que de ellos aprovechan los seres humanos (Chapin, Folke, \& Kofinas, 2009). En un humedal, el componente hídrico es el más visible y más utilizado tanto por los seres humanos, como por la fauna y flora de la región (Perevochtchikova, 2010). De acuerdo con González (2008) los humedales son zonas de gran biodiversidad, que funcionan de recarga para los mantos acuíferos y suelen tener suelos con dificultades de drenaje, lo que permite que se formen cuerpos de agua superficiales, aumentando su tamaño en época de lluvia.

Un sistema socio-ecológico está conformado por componentes físicos tales como el agua, suelo, rocas; organismos vivos como plantas, microbios y animales, incluyendo a los seres humanos. Además, se compone de los productos resultantes de la actividad humana, por ejemplo, alimentos, productos industriales, dinero, contaminación, entre otros (Chapin, et al., 2009). Lo anterior significa que un humedal, como sistema socio-ecológico, se integra por los componentes tangibles y no tangibles. El suelo, agua y la vegetación circundante son los elementos tangibles, mientras que la tenencia de la tierra y las ceremonias en torno al agua, son ejemplos de lo intangible.

En el mundo existen humedales muy famosos como el Pantanal, ubicado en Brasil, Bolivia y Paraguay. La extensión de este sistema es de aproximadamente $210,000 \mathrm{Km}^{2}$, por lo que se le considera como el humedal más grande del mundo (Schlesinger, 2014). En Francia se encuentra el humedal llamado Camarga (Camargue en francés), el cual es reconocido por la presencia de Flamingos rosas, así como de otras 237 especies de aves protegidas que sobrevuelan en las 13,000 hectáreas de esta reserva natural (Mediterranean Wetlands, 2014). Alrededor del mundo existen aproximadamente 250 millones de hectáreas que están cubiertas por humedales (Ramsar, 2018).
En México existen importantes humedales como el ubicado en el Valle de Cuatro Ciénegas, en el estado de Coahuila. Esta área contiene poco más de 84,000 hectáreas y desde 1994 fue declarada como Área Natural Protegida; está clasificada como sitio Ramsar, lo cual le dota de importancia internacional. En el caso de Cuatro Ciénegas tanto la agricultura como el turismo inciden de forma negativa en la conservación de la biodiversidad (Ortiz \& Romo, 2016). De acuerdo con el sitio web de la Convención sobre los Humedales o Convención Ramsar, en México son 142 los sitios clasificados con importancia internacional.

Para la Convención Ramsar, un humedal es una:

(...) extensión de marismas, pantanos y turberas, o superficies cubiertas de agua, sean éstas de régimen natural o artificial, permanentes o temporales, estancadas o corrientes, dulces, salobres o saladas, incluidas las extensiones de agua marina cuya profundidad en marea baja no exceda de seis metros (Convención Ramsar, 2016)

Para esta convención existen humedales que se pueden clasificar en: 1) marinos, 2) estuarinos, 3) lacustres, 4) ribereńos, 5) palustres y 6) artificiales. En México, la Convención Ramsar entró en vigor el 4 de noviembre de 1986 y, actualmente, son 169 países participantes.

Una de las razones más importantes para conservar los humedales es porque son sumamente biodiversos: proveen de sustento a diferentes especies de aves, mamíferos, reptiles, anfibios, peces e invertebrados. También permiten sostener actividades agropecuarias como la siembra de arroz o maíz y la cría de diferentes tipos de ganado. El agua almacenada puede ser aprovechada tanto para el uso doméstico de las poblaciones aledañas como para el uso industrial. Por todo lo anterior, los humedales, como sistemas socio-ecológicos, requieren ser estudiados desde diversos ángulos, con la finalidad de entender las bases de su sostenibilidad y así ayudar a su preservación. 
A pesar de la evidente importancia por conservar los humedales, la realidad es que existen procesos y fenómenos que atentan contra estos sistemas socio-ecológicos. Por ejemplo, el interés por aumentar las zonas cultivables ha propiciado la desecación de grandes cantidades de hectáreas. En el estado de Tlaxcala, los humedales se utilizaron para fines agrícolas y ganaderos, además, fueron objeto de desecación y, sus aguas fueron desviadas para la conformación del Distrito de Riego 56 AtoyacZahuapan. En esta zona, los humedales fueron transformados en "calales": un sistema agrícola de origen prehispánico que se practica en zonas anegadizas o pantanosas (González, 2008).

En la época prehispánica, los humanos que vivían en la región de los humedales se asentaban en las partes más elevadas, solían drenarlos para cultivar, colectar plantas y peces o cazar aves para el consumo familiar (González, 2003). A lo largo del siglo $\mathrm{xx}$, diversas lagunas de Tlaxcala fueron afectadas por los proyectos de desecación. La Laguna de Acuitlapilco comenzó a desecarse a finales del siglo XIX para aumentar las tierras de cultivo, pero también por el azolve del lecho lacustre, el agotamiento de los manantiales, la disminución de las lluvias y por la deforestación de La Malinche (Rodríguez, 2013).

De acuerdo con Gonzalez (2008), las áreas desecadas fueron utilizadas hasta la década de 1980 para la siembra de árboles frutales como capulín, chabacano, tejocote, durazno y zapote blanco, así como maíz, haba, chícharo, cilantro y flores comerciales como gladiolo y nube. Con la desecación de la laguna se perdieron los camellones y las chinampas ubicadas a los márgenes de la laguna. En el lugar que se encontraban el agua ahora hay cultivos de maíz y viviendas familiares que suelen inundarse en años lluviosos. La desecación también afectó a otros poblados que utilizaban el agua para sus cultivos (Moctezuma, 2013).

La historia de la laguna de Acuitlapilco es similar a otras historias en México. Por ejemplo, la cuenca del Alto Lerma, ubicada en la región de Toluca, Atlacomulco e Ixtlahuaca, en el Estado de México. Las desecaciones para el uso agrícola, la creación de caminos y carreteras, así como para aprovechar los suelos para la instalación de industrias y zonas habitacionales han propiciado lo que González y Velasco (2015) denominan como "la muerte de un humedal". Este tipo de historias demuestran la importancia de seguir estudiando los humedales con la finalidad de contribuir a su mayor entendimiento, difusión y preservación.

Con base en esto, el principal objetivo de este artículo es analizar la historia de un humedal artificial localizado en una comunidad indígena del Estado de México. Para llevar a cabo dicho objetivo se reconstruye el tipo de participación de actores específicos, en este caso una fundación filantrópica y un grupo de indígenas mazahuas que habitan en Chotejé, municipio de San Felipe del Progreso. La relación entre actores es mediada, por la intención de generar proyectos de desarrollo con impacto benéfico a la comunidad. Este artículo describe estas relaciones y, su impacto en la creación de un humedal artificial comunitario.

\section{Zona de estudio}

San Felipe del Progreso es uno de los dieciocho municipios que conforman la Región V Atlacomulco. Su principal característica ambiental, y de sus alrededores, es su clima templado sub-húmedo, con un rango de temperatura de 10 y $14^{\circ} \mathrm{C}$. La altitud mínima es de 2,500 msnm y máximo de 3,200. La precipitación promedio anual es de $842.2 \mathrm{~mm}$, con lluvias abundantes de julio a septiembre y sequía de diciembre a abril. Este municipio pertenece a la cuenca del río Lerma-Toluca, en el centro de su territorio se encuentra la Presa Tepeltitlán, la cual irriga las tierras agrícolas (INEGI, 2009).

Una segunda característica de San Felipe del Progreso es que es habitado por uno de los cinco grupos indígenas originarios del Estado de México: los mazahuas. Este grupo es el más numeroso dentro 
del estado. Los mazahuas habitan en quince municipios y, de acuerdo con el Consejo Estatal para el Desarrollo Integral de los Pueblos Indígenas (CEDIPIEM), la mayor concentración ocurre en los municipios de San Felipe del Progreso, Ixtlahuaca y Atlacomulco. La población de tres ańos y más, que es hablante de lengua mazahua, asciende a 116,240 individuos, de acuerdo con el último censo levantado por el INEGI (2010).

Como tercera característica, el CEDIPIEM reporta que la población mazahua se dedica principalmente a la agricultura. De manera complementaria a la elaboración y venta de artesanías. Asimismo, recibe remesas, producto de la migración nacional e internacional. Lo anterior corresponde con lo planteado por Guadarrama, Vizcarra, \& Lutz (2009), quienes describen que la migración entre las comunidades mazahuas del norte del estado son el resultado del agotamiento de las tierras fértiles, la ausencia de empleo, necesidades económicas insatisfechas, carencias de bienes materiales para la subsitencia y, sobre todo, ausencia de políticas que impulsen y sostengan la producción del sector campesino.

La comunidad de Chotejé se localiza en el municipio de San Felipe del Progreso, en las coordenadas longitud -100.020833, latitud 19.723889, a 2,821 msnm. Para llegar a Chotejé desde la ciudad de Toluca se recorren $73 \mathrm{~km}$. La comunidad tiene una población total de 1,454 habitantes, de los cuales 683 son hombres y 771 mujeres (INEGI, 2010). El $40.96 \%$ de los habitantes habla mazahua. Las oportunidades laborales se limitan a las actividades del campo, algunos habitantes buscan trabajo en la Ciudad de México, en la construcción, mercados y central de abastos; en proporciones menores migran a Estados Unidos de América.

Figura 1. Mapa de ubicación de San Felipe del Progreso. Fuente: CNDI (2006)

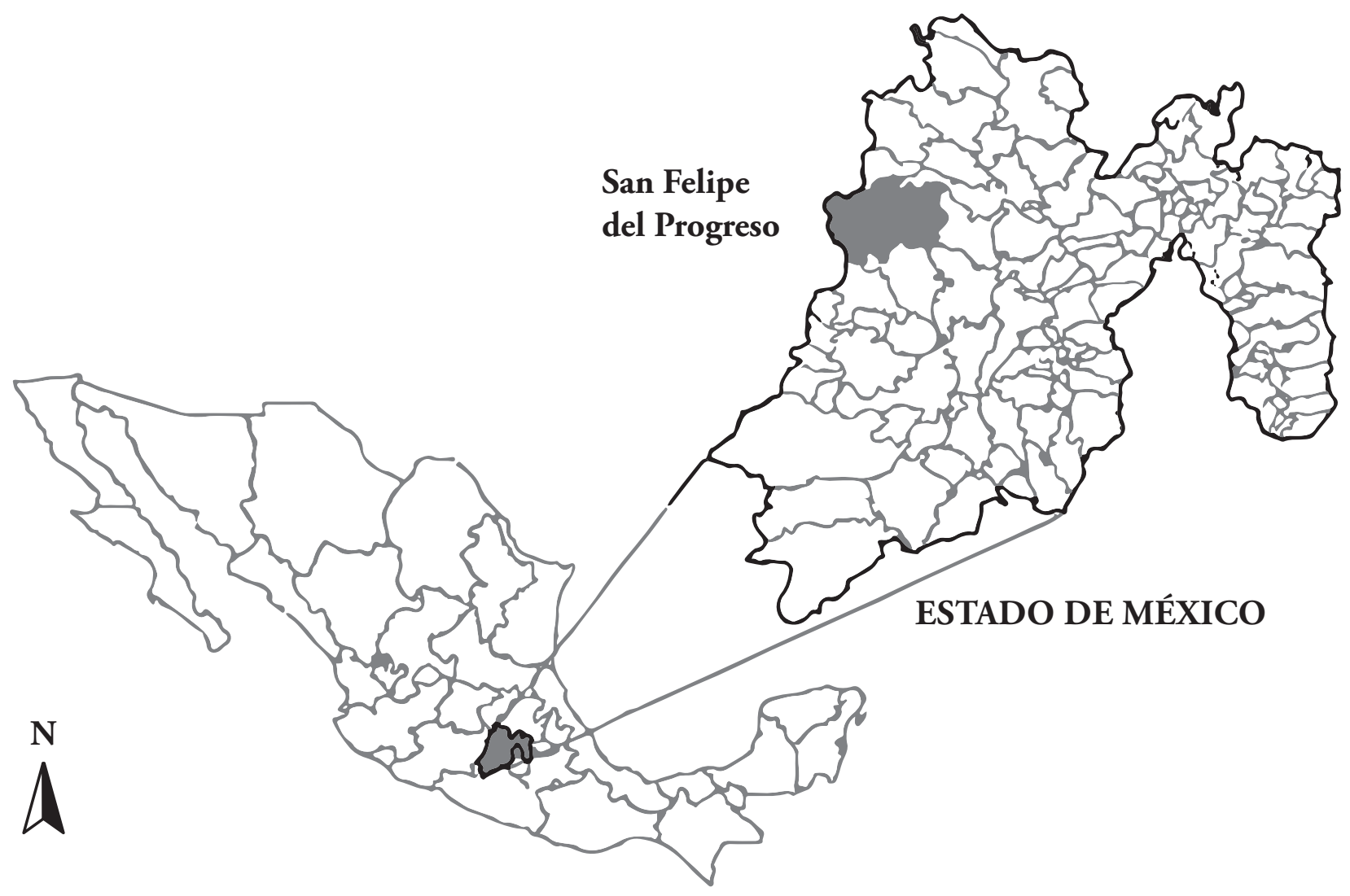

38 Ciencia, Ambiente y Clima 2019; 2(1): 35-43 • Artículos originales 


\section{Metodología}

Para llevar a cabo la investigación que sustenta este artículo se utilizó una metodología cualitativa (Tarrés, 2015). Se realizó un recorrido de área por el municipio de San Felipe del Progreso con la finalidad de detectar las comunidades que tuvieran en su territorio un humedal artificial. El resultado de este recorrido fue la identificación de la localidad de Chotejé y de su humedal. Luego, se llevaron a cabo pláticas informales con algunos habitantes para recabar información sobre el humedal y sobre posibles informantes claves. Dichas conversaciones dieron como resultado el permiso necesario para realizar la investigación.

Posteriormente, se aplicó la técnica de bola de nieve con diversos habitantes de Chotejé. Así, fue posible entablar comunicación con una respetada mujer de la comunidad que participa activamente en proyectos de desarrollo gestionados por una fundación de corte filantrópico. El papel de la informante clave fue fundamental para servir como portera en la comunidad y así ubicar a más habitantes que son usuarios del humedal artificial y que han sido beneficiarios de diversos proyectos sociales y económicos. Por último, se realizaron entrevistas a diversos informantes claves con el propósito de reconstruir la historia del humedal artificial.

\section{Resultados}

En Chotejé se observan diversas obras y prácticas orientadas a la conservación del suelo y el agua. Por ejemplo, la comunidad cuenta con una presa de concreto para captación del agua de lluvia para las actividades productivas. Esta obra es el resultado de la participación comunitaria dentro del programa de Conservación y Uso Sustentable de Suelo y Agua (COUSSA), el cual tiene como finalidad fortalecer los recursos productivos primarios, como el suelo, agua y la vegetación, mediante un uso y manejo sustentable, que a su vez repercuta en la seguridad alimentaria de las familias y en su reducción de pobreza.
Para el caso de Chotejé, este programa apoya a productores rurales que no cuentan con infraestructura suficiente para el almacenamiento de agua con fines domésticos, o para llevar a cabo actividades agropecuarias. Además, se cuenta con el apoyo de la Comisión Nacional de las Zonas Áridas (CONAZA), que a través de la Secretaría de Agricultura, Ganadería, Desarrollo Rural, Pesca y Alimentación (SAGARPA) y el Gobierno del Estado de México, las Agencias de Desarrollo Rural (ADRS), establecen zonas vulnerables y aptas para recibir beneficios. A lo anterior, se añade la intervención de la Fundación Pro Mazahua como Agencia de Desarrollo Rural.

El Patronato Pro Mazahua fue constituido en 1997, una asociación civil cuyo objetivo ha sido y sigue siendo propiciar el desarrollo en favor de la inclusión social y la lucha contra la pobreza que existe entre el grupo mazahua del Estado de México. Este patronato comenzó su trabajo principalmente en las comunidades de San Felipe del Progreso y San José del Rincón. Durante el 2009 se origina la Fundación Pro México Indígena, con el objetivo de impulsar el surgimiento de los pueblos originarios de México, utilizando un modelo de desarrollo integral y sustentable que está basado en cuatro ejes estratégicos.

El primero de ellos es el eje físico y ambiental, bajo el cual se respeta la capacidad de renovación, reutilización y jerarquización del aprovechamiento de los recursos naturales. El segundo eje corresponde al elemento social y humano, que integra la salud, la seguridad alimentaria y la vivienda rural sustentable, mediante talleres de organización y habilidades para el desarrollo humano integral. El tercer eje enfatiza el aspecto económico y productivo, orientado al desarrollo de capacidades locales, que generen ingresos o formen cadenas de valor productivas. El último eje, cívico y cultural, pretende revalorizar y rescatar la cultura de los pueblos indígenas.

Lo expuesto hasta este momento denota el interés por diversos actores externos a la comunidad de 
gestionar y otorgar apoyos comunitarios bajo un modelo que privilegia la sustentabilidad ecológica, social y económica. Sin embargo, estos proyectos requieren siempre de la activa participación de las comunidades beneficiarias. Para el caso que nos ocupa, una informante clave recuerda que a finales de la década de 1990 el comité Pro Mazahua se reunió con los delegados de Chotejé, con la finalidad de ubicar las principales necesidades de la población y las posibles vías de acción en las que dicha fundación podría participar.

El primer proyecto que realizó la fundación fue colocar piedras de mampostería para evitar los deslaves de la tierra. Este proyecto detonó la confianza de la población mazahua hacia la fundación. A su vez, esta tiene como principio apoyar los proyectos que surgen desde la comunidad. Así, a poco más de dos décadas de relación, se han concretizado proyectos de estufas ecológicas, micro túneles, programas de reforestación, gallineros para obtención de huevo y carne, criaderos de guajolotes, purificadoras de agua, la construcción de un centro de salud y, para el objetivo central de este artículo, una presa captadora de agua.

Actualmente, dieciséis mazahuas integran el grupo que trabaja con la fundación en diferentes proyectos, incluyendo la presa. Al costado de la válvula de agua de la presa existe una placa fechada el $12 \mathrm{de}$ mayo de 2010 que dice:

Con esta fecha se inauguran las obras de conservación de suelo y agua en la localidad de Chotejé, mismas que son una invitación a que sigamos trabajando unidos y con responsabilidad compartida entre comunidades y las autoridades de los diferentes niveles de gobierno en la implementación de acciones que propicien el desarrollo de las comunidades y la conservación del medio ambiente.

La presa capta el agua de lluvia y provee el riego durante la época de secas a un invernadero que es trabajado por cuatro personas de diferentes familias. Lo anterior denota la importancia de la colectividad, tal como lo apunta Morales (2000:14), "(...) muchas de las actividades en las que toda la población de determinados lugares debe tomar parte, son consultadas, consensadas y comunicadas con un sentido verdaderamente comunitario". Estas actividades y decisiones son determinadas por individuos que gozan de prestigio comunitario, el cual es acumulado por ańos social y laboralmente, y no está relacionado con la situación económica individual.

Este humedal artificial ha generado diversos beneficios tangibles e intangibles entre quienes lo manejan. Por ejemplo, el invernadero produce jitomate para el comercio, principalmente entre julio y noviembre. Los ingresos que se obtienen con la venta permiten complementar las estrategias de sustento de la población, que se dedica a la agricultura mediante el sistema agrícola tradicional de milpa y a la cría y engorda de ganado vacuno y porcino. En palabras de uno de los beneficiarios de la utilización del agua para el invernadero se denota el interés por aprender sobre nuevos cultivos y sus potencialidades para comercializarlos:

(...) al principio nos costó mucho trabajo entender cómo debía ser el manejo del invernadero, y aunque venía el técnico y nos explicaba, no sabíamos por qué no teníamos buena cosecha. Estuvimos casi como dos años sin producir nada, o sólo para comer, ya hasta después entendimos cómo es el procedimiento para el cuidado de las plantas. Ahora hemos podido vender un aproximado de cinco toneladas en buena época de cosecha desde hace casi tres años (Juan Pérez, Comunicación personal, diciembre 2018 ${ }^{1}$ )

Este informante clave reconoce que el empeño y trabajo que han puesto las familias que operan el invernadero les ha permitido tener cosechas

1. Para proteger la confidencialidad del informante clave se omite su nombre y apellido real y se sustituye por un nombre ficticio. 
constantes y, por lo tanto, clientes fijos que llegan directamente al invernadero para comprar la cosecha. De esta forma, el grupo de mazahuas encargado del proyecto no requiere transportarse para comercializar parte de la cosecha hacia diversos mercados regionales. Otros productos que suelen cultivar son: nabos, cilantro, brócoli, col, coliflor, verdolagas, rábanos, zanahorias, cebollas, chiles, quelites y nopales. Estos productos aportan a la seguridad alimentaria de las familias propietarias del invernadero, así como a las de Chotejé.

Una vez al mes los productos son llevados a un tianguis saludable, realizado dentro de Chotejé. Este espacio se propicia el trueque entre vecinos; a cambio de los cultivos se acepta leche, huevo, carne, frutas, maíz o cualquier otro producto alimenticio que las familias necesiten. Durante las entrevistas realizadas a la población involucrada en el invernadero y en el tianguis saludable se exalta la importancia de tener alimentos saludables, libres de químicos, así como la conservación de la alimentación que los abuelos y padres han enseñado. Recientemente, en estos tianguis, ha hecho su aparición la comercialización de cempasúchil y stevia.

Una de las condiciones que están establecidas para ser acreedor de los beneficios que otorga SAGARPA, en lo referente al agua que se capta en el bordo artificial, es la integralidad "(...) alrededor de la cual se vinculen las obras complementarias para su adecuado funcionamiento, y conforme al territorio atendido, el desarrollo del resto de obras y prácticas de Conservación y uso sustentable de suelo y agua" (SAGARPA, 2009:5). Por lo anterior, el agua no solo se utiliza para el invernadero, también dota agua a la escuela primaria Profesor Carlos Hank González y a la Telesecundaria 0414 Justo Sierra.

Ambas escuelas se localizan a 750 metros del humedal. Esta dotación de agua beneficia la higiene y salud del estudiantado. Si bien la producción de alimentos y uso humano son los principales beneficios que se obtienen del agua captada, se tiene previsto utilizar filtros para su purificación y consumo humano. Esta situación es considerada como urgente por la población, ya que en ocasiones la comunidad tiene dificultades para conseguir agua potable, problemática que ha generado un elevado consumo de bebidas azucaradas, con el riesgo que ello implica para salud de la población y la generación de basura en la comunidad.

\section{Discusión}

Durante mucho tiempo se ha discutido sobre los problemas que enfrentan las comunidades rurales para generar procesos de desarrollo local. Inclusive, se ha criticado el papel de las instituciones gubernamentales y la ausencia de políticas eficientes para atender los problemas del medio rural (Moctezuma \& Murguía, 2014). Sin embargo, Chotejé es un ejemplo del papel que juegan los agentes de desarrollo externos para disminuir las carencias que aquejan a zonas vulnerables. Resulta pertinente indagar las condiciones que propician una intervención exitosa por parte de las fundaciones en las comunidades indígenas. A continuación, se mencionan tres que son manifiestas en Chotejé.

Una primera condición es la colectividad, puesto que en las zonas rurales las posibilidades de acceder a créditos o proyectos se ven cortadas por la limitada posesión de tierras, infraestructura, dinero, mano de obra y otros factores. Sin embargo, la organización entre grupos vecinales con un objetivo común es una característica esencial para generar resiliencia comunitaria. En el caso de la comunidad mazahua de Chotejé, el ceder un espacio común para la construcción del humedal y el invernadero, así como el compromiso para trabajar de manera conjunta, es lo que ha permitido acceder a diferentes recursos y generar opciones productivas.

La segunda condición es la capacitación, que no necesariamente se refiere a adquirir conocimientos o destreza física, también integra temas como visión a largo plazo, proyectos integrales e inclusivos, o la importancia de gestionar proyectos endógenos. La 
visión a largo plazo favorece cambiar el paradigma de que todo proyecto es viable solo si se ofrecen beneficios a corto plazo. Los proyectos integrales e inclusivos posibilitan el aprovechamiento de recursos que ya se tienen, se relacionan con la visión a largo plazo y permiten aprovechar las distintas características de los integrantes en los proyectos, teniendo como resultado el fortalecimiento del grupo de trabajo.

Por último, está la importancia de que los proyectos deben gestarse desde dentro de la comunidad, pues no hay quien conozca más de las necesidades, prioridades y alcances de un grupo de trabajo que quienes viven el día a día en una comunidad. En el caso de Chotejé se generó una alternativa de desarrollo, lo que permite acabar con la aseveración de que las comunidades no alcanzan el desarrollo porque viven en un constante estado de conformismo o apatía, en donde la asistencia se acepta solo cuando no implique esfuerzo alguno o tiempo de espera para obtener los beneficios esperados.

Resulta complicado diseñar estrategias de desarrollo y que estas se puedan replicar, debido a que cada territorio cuenta con recursos específicos, problemáticas y objetivos distintos, sin embargo, una correcta intervención por parte de las instituciones es lo que puede promover el progreso tan ansiado para las comunidades vulnerables. Para ello, es necesario que los actores propiciadores del desarrollo trabajen en generar la confianza necesaria y suficiente con la gente que se pretende sea beneficiaria; asimismo, en entender la organización y estructura de la comunidad, lo cual a su vez implica el respeto y comprensión de la cultura (Uphoff, 1995).

\section{Conclusiones}

Gracias al constante trabajo con las comunidades se puede concluir que el papel de las organizaciones que buscan el desarrollo comunitario se considera fundamental, siempre y cuando respeten las características del territorio, sean culturales, de disponibilidad de recursos, medioambientales, entre otras. El plantear objetivos en común, tales como propiciar mejores condiciones en pro de la seguridad alimentaria, la generación de proyectos productivos que intentan disminuir la migración laboral o contar con los medios necesarios para la producción de alimentos son solo algunas de las líneas de acción que en la comunidad de Chotejé han dado resultados positivos para diversos grupos.

A pesar de lo anterior, se considera importante también que la gente que se involucre en los proyectos, respecto a la toma de decisiones, sea capaz de coordinar con un sentido altruista y de cooperativismo, con gran capacidad de escucha de las opiniones de los involucrados en los proyectos. De este modo sabrá aprovechar las capacidades y potencialidades de los involucrados. Es por tanto una oportunidad de mostrar que el desarrollo se planea desde dentro, y no solo adaptando las circunstancias a los requerimientos impuestos por un estrato con tintes de rigidez.

\section{Agradecimientos}

Este artículo es resultado del proyecto "Valoración ambiental, social y productiva de los humedales interiores artificiales de México", financiado por el Consejo Nacional de Ciencia y Tecnología de México (CONACyT). Los autores agradecen el apoyo de la Universidad Autónoma del Estado de México para la realización de la investigación, así como del CONACyT para la financiación del proyecto.

\section{Referencias}

Chapin, F. S., Folke, C. \& Kofinas, G. (2009). A Framework for Understanding Change. En Chapin F., G. Kofinas, \& C. Folke. (Eds.), Principles of Ecosystem Stewardship (pp. 3-28). New York, EE. UU: Springer.

Convención Ramsar. (2016) 
Gonzalez, A. (2003). Cultura y agricultura: transformaciones en el agro mexicano. Ciudad de México, México: Universidad Iberoamericana.

González, A. (2008). Humedales en el suroeste de Tlaxcala. Agua y agricultura en el siglo XX. Ciudad de México, México: Universidad Iberoamericana.

González, A. y Velasco J. J. (2015). La muerte de un humedal. Ambiente y cultura en la Cuencia alta del Río Lerma. Ciudad de México, México: Alpe Ediciones.

Guadarrama, X., Vizcarra, I., \& Lutz, B. (2009). De la migración: ausencias masculinas y reacciones femeninas mazahuas. Relaciones. Estudios de Historia y Sociedad, 30(118): 183-219. Recuperado de: http://www.redalyc.org/articulo.oa?id $=13712904007$

Instituto Nacional de Estadística y Geografía (INEGI). (2009). Prontuario de información geográfica municipal de los Estados Unidos Mexicanos. San Felipe del Progreso, México. 2009. México, México: Instituto Nacional de Estadística y Geografía.

Instituto Nacional de Estadística y Geografía (INEGI). (2010). Censo de Población y Vivienda 2010. México, México: Instituto Nacional de Estadística y Geografía.

Mediterranean Wetlands. (2014). Biodiversity in Mediterranean Wetlands. A Status Report at the Beginning of the 21 Century. Arles, Francia: Mediterranean Wetlands Observatory.

Moctezuma, S. (2013). Cambios y continuidades en el manejo de huertos familiares del suroeste de Tlaxcala, México, en: Perspectivas Latinoamericanas, 10(1): 83-101. Recuperado de: https://nanzan-u.repo.nii.ac.jp

Moctezuma, S., \& Murguía, V. (2014). Estrategias de subsistencia en tres sociedades rurales de México. Perspectivas Latinoamericanas, 11(1): 112-126. Recuperado de: https://nanzan-u. repo.nii.ac.jp
Morales, E. S. (2000). El sabor agrio en la cultura mazahua. Toluca, México: Instituto Mexiquense de Cultura.

Ortiz, S. E., \& Romo, M. L. (2016). Impactos socioambientales de la gestión del agua en el área natural protegida de Cuatro Ciénegas, Coahuila. Región y Sociedad, 28(66): 195-230.

Perevochtchikova, M. (2010). “La problemática del agua: revisión de la situación actual desde una perspectiva ambiental", en: Lezama, J. L y Graizbord B. (2010). Los grandes problemas de México. Medio ambiente. Ciudad de México, México: El Colegio de México, pp. 61-104.

Ramsar. (2018). Global Wetland Outlook. State of the world's wetlands and their services to people 2018. Gland, Switzerland: Ramsar Convention Secretariat.

Rodríguez, E. (2013). Acuitlapilco. Cambio cultural y crisis. Ciudad de México, México: Secretaría de Educación Pública del Estado de Tlaxcala.

Secretaría de Agricultura, Ganadería, Desarrollo Rural, Pesca y alimentación (SAGARPA). (2009). Lineamientos operativos especificos para la aplicación de recursos del componente de conservación y uso sustentable de sueño y agua, del programa de uso sustentable de recursos naturales para la producción primaria, en torno a la estrategia PESA (COUSSA-PESA). México, México: SAGARPA.

Schlesinger, S. (2014). The whole Pantanal, not just the half. Soy, waterway and other threats to the integrity of the Pantanal. Mato Grosso, Brasil: Ecosystem Alliance.

Tarres, M. (Coord.) (2015). Observar, escuchar y comprender. Sobre la tradición cualitativa en la investigación social. Ciudad de México, México: El Colegio de México A.C., Flacso. Uphoff, N. (1995). "Adaptar los proyectos a la gente", en: Cerna, M. (Coord.). Primero la gente. Variables sociológicas en el desarrollo rural. Ciudad de México, México: Fondo de Cultura Económica. Pp. 535-581. 